\title{
Berthold Weiß
}

\section{Die Onleihe in einer wissenschaftlichen Bibliothek am Beispiel Fulda}

Zusammenfassung: Die Hochschul- und Landesbibliothek Fulda (HLB Fulda) bietet als wissenschaftliche Bibliothek mit der Onleihe ein Angebot an elektronischen Medien an, welches eher als ein Serviceangebot für öffentliche Bibliotheken angesehen wird. Es wird aufgezeigt, warum sich die HLB Fulda auf diesem Gebiet engagiert und dabei kurz die besondere Situation in Fulda angerissen. Die Einführung der Onleihe und die Etablierung des Angebotes werden neben einem Ausblick auf die weitere Entwicklung dieser Dienstleistung in Fulda dargestellt.

Schlüsselwörter: Digitale Bibliothek „Onleihe“, Wissenschaftliche Bibliothek, Öffentliche Bibliothek

\section{The e-book library service "Onleihe” in the University and State Library in Fulda}

Abstract: As an academic and research library the University and State Library of Fulda (Fulda HLB) offers a range of electronic media via the library service "Onleihe" which, however, is usually looked at as a service with a leaning towards public libraries. The article shows why HLB Fulda has got involved in this field thereby also touching upon the specific situation in Fulda. The introduction as well as the establishment of the e-book service "Onleihe" are outlined and the prospects of this service are also looked at.

Keywords: digital library “Onleihe”, academic library, public library

Berthold Weiß: berthold.weiss@hlb.hs-fulda.de

\section{Einleitung}

Die Hochschul- und Landesbibliothek Fulda (HLB Fulda) hat eine über 235-jährige Tradition als wissenschaftliche Bibliothek. Seit rund viereinhalb Jahren bietet sie ihren Nutzerinnen und Nutzern mit der Onleihe ein Angebot an elektronischen Medien an, welches zunächst als Dienstleistung für öffentliche Bibliotheken angesehen wird. Warum engagiert sich eine wissenschaftliche Bibliothek auf 
diesem Sektor? Was ist die Idee, welches ist der Nutzen und für wen rechnet sich der Aufwand? Ist das nur für Fulda interessant oder auch für andere wissenschaftliche Bibliotheken? Im Folgenden wird versucht, diese Fragen zu beantworten. Zunächst ist dafür ein Gang in die jüngere Geschichte der HLB Fulda notwendig.

Die heutige HLB Fulda wurde im Jahre 1778 von Fürstbischof Heinrich von Bibra als „öffentliche“ Bibliothek gegründet. Die Zuständigkeiten wechselten mehrfach bis sie schließlich zum Land Hessen übergingen. Als nächster Meilenstein ist für den hiesigen Beitrag das Jahr $2001 \mathrm{zu}$ erwähnen, in dem die selbstständige Hessische Landesbibliothek in die damalige Fachhochschule Fulda ${ }^{1}$ integriert wurde und nun als Hochschul- und Landesbibliothek fungierte. ${ }^{2}$

Durch diesen Vertrag war ihr Fortbestehen als Landesbibliothek gesichert und es konnte ein Ausbau von Dienstleistungen und Serviceangeboten erfolgen. Dazu zählten - wenn auch nur als kleiner Schwerpunkt - die stadtbibliothekarischen Aufgaben, die sie traditionsgemäß schon seit ihrer Zeit als Hessische Landesbibliothek wahrnahm. Dies ist eine Fuldische Besonderheit, da es in Fulda keine „klassische“ öffentliche Bibliothek gibt, sondern die Aufgaben verteilt sind: Bis zum Jahre 2011 existierte mit der Centralbücherei des Büchereiverbundes Fulda e. V. eine „Familienbibliothek“, die vor allem den Schwerpunkt des Kinder- und Jugendbereiches in Fulda abdeckte. Einführende Sachliteratur und ein belletristisches Angebot fand man dort zwar auch, aber das Angebot war kleiner als das der HLB Fulda am Innenstadt-Standort Heinrich-von-Bibra-Platz.

Im stadtbibliothekarischen Bereich richtete man die Aufmerksamkeit nun auf das elektronische Angebot; 2004 wurden erstmals 100 Hörbücher angeschafft. Dabei handelte es sich hauptsächlich um Titel aus dem Bereich aktueller Belletristik, aber auch „Klassiker“ und Sachliteratur wurden zur Ausleihe angeboten. Das Angebot wurde so stürmisch angenommen, vielleicht auch aufgrund der Presseresonanz ${ }^{3}$, dass nach wenigen Tagen die Hörbücher alle ausgeliehen waren. Drei Monate später wurde der Bestand dann auf rund 250 Titel ausgebaut. ${ }^{4}$

1 Im Jahre 2006 erfolgte die Umbenennung in „Hochschule Fulda - University of Applied Sciences“.

2 Vertrag zwischen dem Land Hessen, der Fachhochschule Fulda und der Stadt Fulda über die Weiterführung von Funktionen der Hessischen Landesbibliothek vom 4.10.2010, siehe auch ausführlich dazu sowie zur wechselvollen Geschichte der HLB Fulda: Riethmüller, Marianne: Eine Bibliothek im Wandel: Die neue kombinierte Hochschul-, Landes- und Stadtbibliothek Fulda. In: ABI-Technik 31 (2011), 4, S. 192-197.

3 Hörbücher in der Landesbibliothek. In: Fuldaer Zeitung, 5.2.2004; der Hessische Rundfunk brachte sogar ein Interview mit dem stellvertretenden Bibliotheksleiter der HLB Fulda.

4 Weitere Hörbücher in der Bibliothek. In: Fuldaer Zeitung, 21.5.2004. 100 weitere Hörbücher. Landesbibliothek erweitert ihren Bestand. In: Marktkorb. Fuldaer Bote - das Wochenblatt für 
Die Nachfrage war also geweckt - warum dann nicht auch ein Angebot an digitalen Medien für die stadtbibliothekarischen Nutzerinnen und Nutzer offerieren? Der wissenschaftliche Bereich war ja über die Beteiligung an Konsortien, v. a. am HeBIS-Konsortium, bestens mit elektronischen Zeitschriften, Datenbanken und E-Books abgedeckt. So wurde also die Markteinführung der Onleihe im Jahre 2007 mit Interesse beobachtet und zwei Jahre später stieg man in der HLB Fulda auch ein.

\section{Phase I: Onleihe in Fulda von 2009-2013}

Im Mai 2007 eröffneten die öffentlichen Bibliotheken in Hamburg, Köln, München und Würzburg gemeinsam mit der DiViBib GmbH aus Wiesbaden den neuen Service „Onleihe“, der den jeweiligen Nutzerinnen und Nutzern ermöglichte, digitale Medien über die Homepage ihrer zuständigen Stadtbibliothek rund um die Uhr auszuleihen. ${ }^{5}$

Die HLB Fulda verfolgte diese Entwicklung mit Interesse in der Absicht, für ihre Nutzerinnen und Nutzer auch hier aktiv zu werden. 2009 wurde mit der Firma DiViBib Kontakt aufgenommen und man erhielt im Juli ein Angebot zur Einrichtung und zum Betrieb einer Onleihe sowie zur Lizenzierung eines Medienpakets als Anfangsbestand. Es wurde ein Arbeits- und Zeitplan erstellt, der in den nächsten fünf Monaten abgearbeitet wurde. Neben der Klärung vertrags- und datenschutzrechtlicher Fragestellungen musste vor allem die technische Schnittstelle realisiert werden. So war eine Verbindung zum ous-Modul des Bibliothekssystems PICA notwendig. Die „Ausleihen“ werden zwar nicht im bibliothekseigenen Ausleihsystem abgewickelt, sondern über die Server der Firma DiViBib, dennoch musste ein Abgleich zwischen Leseausweis und Passwort erfolgen, um überhaupt zur Ausleihe zugelassen zu werden. Weiterhin wird vor dem Download auch überprüft, ob bei der Nutzerin oder dem Nutzer noch ausstehende Gebühren auf dem „klassischen“ Konto vermerkt sind. In diesem Fall ist eine Ausleihe nicht möglich. Auch die Metadaten der Titel mussten in den Online-Katalog eingespielt werden, um die Onleihe-Medien nicht nur über das für Fulda realisierte Webportal aufrufen zu können, sondern auch - wie alle verfügbaren Medien - über eine Recherche im Online-Katalog finden und per Link direkt nutzen zu können. Dies

Osthessen, 19.5.2004; Hörbücher in der Landesbibliothek in Fulda. In: Lauterbacher Anzeiger, 19.5.2004.

5 Öffentliche Bibliotheken in Hamburg, Köln, München und Würzburg ermöglichen Ausleihe digitaler Medien. In: Bibliotheksdienst 41 (2007), 6, S. 674-675. 
war nicht ganz einfach, da nur für die Hälfte der Titel Metadaten im MAB-Format vorhanden waren. So entsprachen die Musiktitel des Naxos-Pakets nicht dem MAB-Standard und wurden daher auch nicht übernommen. ${ }^{6}$

Am 8. Dezember 2009 war es dann soweit: Die HLB Fulda startete als erste wissenschaftliche Bibliothek eine Onleihe. ${ }^{7}$ Dass dies auch für den Anbieter ein wichtiges Ereignis war, wurde durch die Anwesenheit von Herrn Hasiewicz, damals bibliothekarischer Direktor der DiViBib GmbH, beim Pressetermin ${ }^{8}$ belegt. Die Onleihe startete in Fulda mit rund 9.400 Titeln und umfasste die Bereiche Belletristik, Musik, Ratgeberliteratur, das „School-Scout-Paket“, welches Unterrichtsmaterialien für Schüler und Lehrer enthielt, Sprachlernbücher sowie den „Spiegel“ im E-Format. Es wurde dann auch gleich das epub-Format zusätzlich zu den bereits gekauften rund 400 E-Books im pdf-Format erworben, damit die Bücher auch mittels E-Book-Reader genutzt werden konnten.

Die Nutzung wurde im ersten Jahr als zufriedenstellend angesehen, auch wenn keine Vergleiche möglich waren, da die HLB Fulda die erste wissenschaftliche Bibliothek mit einem Onleihe-Angebot war und ein Vergleich mit öffentlichen Bibliotheken hinkt: Nach rund zwei Monaten wurden 2.100 Medien ausgeliehen; auf das Online-Portal wurde von 5.200 Nutzerinnen und Nutzern zugegriffen, die insgesamt 77.800 Seiten besuchten - ein weiterer Schritt der HLB Fulda auf dem Weg zur Hybridbibliothek war getan. ${ }^{9}$

Im gesamten ersten Jahr waren 13.300 Besucher aktiv, die rund 7.000 Ausleihen tätigten, davon 3.700 E-Book-Ausleihen. Die Nachfrage war da, denn es wurden 1.150 Reservierungen auf Medien vorgenommen, die gerade verliehen waren. Gründe dafür können sicherlich in den eigenen Werbemaßnahmen gesehen werden. So wurden Handzettel mit den wichtigsten Informationen rund

6 Raatz, Genoveva: Onleihe in der HLB Fulda. In: HeBIScocktail 2010,2; URL: http://www.hebis. de/de/1cocktail/cocktail_detail.php?we_editObject_ID=9334 [Zugriff, wie bei allen folgenden Links, am 13. März 2014].

$7 \mathrm{http} / / /$ www.onleihe.de/fulda.

8 Die Presse berichtete ausführlich, siehe: Bücher, Filme und Musik. Online-Bibliothek. In: Fuldaer Zeitung, 17.12.2009; Bücher, Filme und Musik rund um die Uhr ausleihbar - HLB startet „Onleihe“. In: Osthessen-News, 8.12.2009; URL: http://osthessen-news.de/n1175234/fulda-bcher-filme-und-musik-rund-um-die-uhr-ausleihbar---hlb-startet-onleihe-.html. Bücher, Filme und Musik per Download ausleihen Hochschul- und Landesbibliothek hat ,Onleihe' gestartet. In: Fuldaer Nachrichten/Schöne Nachrichten, 10.12.2009; URL: http://www.schoene-nachrich ten.de/? $\mathrm{p}=47201$. Online leihen. Neues Angebot der Hochschulbibliothek. In: Fulda aktuell, 19./20.12.2009 und 29.12.2009.

9 Erfolg schon nach über zwei Monaten. Die Onleihe der Hochschul- und Landesbibliothek. In: Marktkorb, 7.3.2010. 
um die Onleihe verteilt; ebenso wurde im monatlich erscheinenden Newsletter ${ }^{10}$ auf ausgewählte Neuerscheinungen hingewiesen und die Onleihe wurde in das Schulungsangebot, welches über die beiden Volkshochschulen der Stadt und des Landkreises Fulda angeboten wurde, aufgenommen. Weitere Vorteile waren, dass keine Mahngebühren entstanden, da die Medien automatisch wieder abgegeben wurden und wie beim herkömmlichen Medienangebot konnten auch hier fünf Medien kostenfrei reserviert werden, die dann zwei Tage bereitgehalten wurden.

Allerdings gab es auch Schattenseiten: Das Naxos-Musikpaket, welches Klassik, Blues und Jazz umfasste, wurde nie richtig angenommen. Im Jahre 2010 gab es 453 Ausleihen im Bereich E-Musik, 2011 waren es nur noch 127 Ausleihen. Folglich wurde dieses Angebot, für das jährlich extra Kosten entstanden, Ende 2011 wieder gekündigt. Die Besucherzahlen gingen schon im Jahre 2011 zurück und lagen auch 2012 nur noch bei 9.300 Besuchern. Ebenso stagnierten die Ausleihzahlen in gleicher Höhe wie im ersten Jahr. Was waren die Gründe? Vielleicht war eine Sättigung vorhanden, da die Besucher die Onleihe kannten. Der Hauptgrund war wahrscheinlich, dass auch zu wenig neue Titel hinzukamen. Da taten sich mit dem neu gegründeten OnleiheVerbundHessen verbesserte Möglichkeiten auf.

Zwischenzeitlich war die Kooperation mit der Stadt Fulda zur Wahrnehmung stadtbibliothekarischer Aufgaben auf eine völlig neue Stufe gestellt worden. So eröffneten am 9. Oktober 2011 die Staatsministerin des Landes Hessen, Frau Kühne-Hörmann, der Oberbürgermeister der Stadt Fulda, Herr Möller, sowie der Präsident der Hochschule Fulda, Prof. Khakzar, unter Anwesenheit geladener Gäste aus Politik, Wirtschaft und Gesellschaft den Anbau an die Hochschulund Landesbibliothek am Heinrich-von-Bibra-Platz. Die „neue Bibliothek“, das Modellprojekt „Hochschul-, Landes- und Stadtbibliothek Fulda“ (HLSB), ging nun an den Start.

\section{Phase II: Beitritt zum OnleiheVerbundHessen - Onleihe in Fulda ab 2013}

Am 18. Oktober 2010 starteten in Oberursel Staatsministerin Eva Kühne-Hörmann und die Hessische Fachstelle für Öffentliche Bibliotheken den OnleiheVerbundHessen (OVH). Er begann als Zusammenschluss von 17 öffentlichen Bibliothe-

10 Hochschul- und Landesbibliothek Fulda: Newsletter. Fulda 2007. Online unter: http://www. hs-fulda.de/index.php?id=5914. 
ken in Hessen. Ziel war und ist es, vor allem auch kleineren Städten und Kommunen die Möglichkeit zu eröffnen, ein Angebot an digitalen Medien auf- bzw. auszubauen und den Bürgerinnen und Bürgern den unentgeltlichen Zugang zu Online-Medien mit einem ständig steigenden Angebot zu ermöglichen. Die teilnehmenden Bibliotheken verfügten über ein Angebot von rund 12.000 Medien, das regelmäßig aktualisiert wird. Das Projekt wurde zum Start mit 173.500 Euro aus Mitteln des Kommunalen Finanzausgleichs des Landes Hessen gefördert und wird von der Hessischen Fachstelle für Öffentliche Bibliotheken an der (damaligen) Landesbibliothek Wiesbaden koordiniert und betreut. Der OVH startete mit einer Laufzeit von drei Jahren und ist auf Städte mit bis zu 200.000 Einwohnern beschränkt. ${ }^{11}$

Die Stärke des OVH liegt in der Gemeinsamkeit. Als Startkapital für die Erstausstattung einer Einzelbibliothek werden 20.000 Euro empfohlen. Der Verbund startete mit 100.000 Euro. Nur so wird auch eine Medienvielfalt erzielt, die von einer einzelnen Bibliothek nicht vorzuhalten ist. Ebenso sprechen die wesentlich geringeren Betriebskosten für jeden einzelnen Teilnehmer sowie der regelmäßige Neuerwerb in Höhe von fünf Prozent des Erwerbungsetats - rund 30.000 Euro jährlich - für diese Verbundlösung. ${ }^{12}$ Über Arbeitsgruppen in den Bereichen Erwerbung, Statistik, Support und Marketing tauscht man sich untereinander aus. Die Verbundteilnehmer kommen zu regelmäßigen Anwendertreffen zusammen, berichten $\mathrm{u}$. a. über gemeinsame Vorhaben und stimmen über weitere Maßnahmen im Hinblick auf die Onleihe ab. Über einen Mailverteiler wird man regelmäßig mit aktuellen Informationen versorgt.

Mit der nun erfolgten Erweiterung um einen Kinder- und Jugendbereich hin zu einer Hochschul-, Landes- und Stadtbibliothek musste in Fulda folglich auch der nächste Schritt auf dem virtuellen Sektor durch Ausbau des digitalen stadtbibliothekarischen Medienangebotes erfolgen. Schon beim Start des OnleiheVerbundes war die HLB Fulda als interessierter Gast anwesend und beobachtete seitdem die zügige und erfolgreiche Weiterentwicklung des Verbundes.

Intensive Gespräche mit der Hessischen Fachstelle bereiteten den Beitritt vor, dem zunächst von beiden Seiten auch mit Skepsis begegnet wurde. Es gab Fragen und Aussagen wie: Eine wissenschaftliche Bibliothek in einem „öffentlichen“ Verbund? Wobei die wissenschaftliche Bibliothek ja nicht mehr so ganz ausschließlich „wissenschaftlich“ war. Nutzen Fuldaer Studierende den OVH und

11 12.000 Medien: „OnleiheVerbundHessen“ ermöglicht Ausleihe jederzeit und überall. In: Osthessen-News, 27.10.2010. URL: http://osthessen-news.de/n1189127/region-12-000-medienonleiheverbundhessen-erm-glicht-ausleihe-jederzeit-und-berall.html.

12 Ebd. 
nehmen anderen Nutzerinnen und Nutzern die Medien weg? Gibt es nicht auch in den angeschlossenen öffentlichen Bibliotheken Studierende, die dort ihren Ausweis haben und die Medien nutzen? Aber ist es überhaupt wichtig zu unterscheiden, welche „Arten“ von Nutzerinnen und Nutzern das Angebot annehmen? Auch auf Seiten der HLSB gab es Bedenken: Man gibt die eigene Onleihe auf und wird - auch wenn man mitsprechen kann - fremdbestimmt im Hinblick auf Nutzungsbedingungen und Medienerwerb. Die Verhandlungen zogen sich hin ..., aber schließlich trat die HLSB am 1. März 2013 dem OVH, der inzwischen 44 teilnehmende Bibliotheken zählte, bei und erweiterte so ihr Medienangebot auf rund 40.000 Titel.

Auch diesmal mussten wiederum technische Vorarbeiten erfolgen, wie Aktualisierungen der Katalognachweise, denn die Links in den vorhandenen Exemplarsätzen stimmten ja jetzt nicht mehr. Darüber hinaus wurden rund 17.000 Exemplarsätze neu angelegt und es mussten für 6.800 Medien neue Titelsätze erzeugt werden.

Der eigene Onleihe-Webauftritt ${ }^{13}$ wurde durch das OVH-Layout abgelöst und es wurden die gemeinsamen Parameter des OVH übernommen. Demnach können gleichzeitig bis zu 99 Titel entliehen und maximal zehn Titel vorgemerkt werden. Nach einer Benachrichtigung per E-Mail bleiben die Medien drei Tage reserviert. Die Leihfristen betragen für E-Books und E-Audio 14 Tage, für E-Video und E-Musik sieben Tage, für E-Magazine einen Tag und für E-Paper eine Stunde. ${ }^{14}$

Die elektronischen Medien der Onleihe können unter bestimmten Voraussetzungen auch auf mobilen Endgeräten (z. B. eBook-Reader, MP3-Player, Smartphone, Tablet-PC) genutzt werden, sofern es sich um Geräte handelt, die fähig für ein „Digital Rights Management“ sind. Auf den Hilfeseiten des OVH findet man hierzu die Kompatibilitätslisten für die entsprechenden Geräte und Hinweise, wie ein Download erfolgen kann.

Die Gemeinsamkeit bietet Vorteile, weil man nicht mehr für alles selbst zuständig ist. Die Nutzerinnen und Nutzer können Medienwünsche absenden, die zentral bearbeitet werden. Ebenso wird ein Userforum angeboten, in dem die Kunden ihre Erfahrungen mit der Onleihe und deren Anwendung auf mobilen Endgeräten austauschen können. Darüber hinaus ist die HLSB auch der vom Hochschulbibliothekszentrum des Landes NRW betriebenen DigiAuskunft ${ }^{15}$ bei-

13 Der URL http://www.onleihe.de/fulda blieb gleich bzw. lenkt jetzt auf die Verbundseite http://www.onleiheverbundhessen.de/.

14 Leibold, Iris: HLB Fulda erfolgreicher Teilnehmer im OnleiheVerbundHessen. In: HeBIScocktail 2013,3; URL: http://www.hebis.de/de/1cocktail/cocktail_detail.php?we_editObject_ID=9942. 15 http://digiauskunft.digibib.net/onleihe/form.html. 
getreten. DigiAuskunft ist ein kooperatives Auskunftssystem, das die Verwaltung eingehender Anfragen unterstützt. Über diesen Support werden Nutzeranfragen zur Onleihe beantwortet, die nicht über die Hilfethemen gelöst werden konnten.

Die Ausleihzahlen im Bereich der Onleihe haben sich äußerst positiv entwickelt und seit dem Beitritt zum OVH mehr als verdreifacht. Im Jahre 2012 gab es rund 7.000 Ausleihen; ein Jahr später waren es fast 25.000 Downloads. Ein Wermutstropfen für viele: Auf aktuelle Bestseller muss man auch hier „länger“ warten ...

Insgesamt hat sich die Umstellung von der Einzel-Onleihe zum OVH für die HLB Fulda gelohnt, da ein deutlich größeres Angebot an virtuellen Medien auch eine größere Zahl an neuen Nutzerinnen und Nutzern anzieht. Ebenso profitieren die, die sowieso schon Onleihe-Kunden sind, von diesem nun stark erweiterten Angebot. Die regelmäßig abgestimmten Neuanschaffungen lassen das Interesse bei den schon „eingesessenen“ Nutzerinnen und Nutzern nicht sinken.

\section{Fazit und Ausblick}

Für die HLB Fulda war es die richtige Entscheidung, solche Online-Angebote in einer wissenschaftlichen Bibliothek anzubieten. Neben den schon genannten Vorteilen ist auch die Arbeits- und Platzersparnis nicht zu vernachlässigen. Eine Bibliothek benötigt für ein virtuelles Angebot keine zusätzlichen Regalmeter. Ebenso ist die Einarbeitung von Medien weniger aufwändig; ein Aussondern ist nicht mehr nötig. Die leider immer wiederkehrende Diskussion um Leihfristen und Mahngebühren entfällt.

Kunden von Bibliotheken haben unterschiedliche Medienbedürfnisse und wenn wissenschaftliche und nicht-wissenschaftliche Interessen von einer Stelle bedient werden können, dankt das einem nicht nur der einzelne Kunde, sondern es entspricht darüber hinaus bildungspolitischen Empfehlungen, wie sie in bibliotheksstrategischen Papieren schon seit Langem gefordert werden. ${ }^{16}$

Es wird weder bei den Publikationen, die in Printform erscheinen, noch im elektronischen Sektor nachgefragt, ob die gewünschte Information aus dem ,wissenschaftlichen“ oder dem „öffentlichen“ Bibliotheksbereich stammt. Die HLB Fulda hat daher schon immer Wert auf eine breit angelegte Ausstattung mit elek-

16 So zum Beispiel: Bibliothek 2007. Strategiekonzept. Hrsg. von der Bertelsmann Stiftung, Bundesvereinigung Deutscher Bibliotheksverbände e. V. Gütersloh 2004, S. 21. Online unter: http:// www.bideutschland.de/download/file/bibliothek_2007/strategiekonzept_langfassung.pdf. 
tronischen Veröffentlichungen gelegt. Neben dem Erwerb elektronischer Fachinformationen, v. a. für die Fachbereiche und Studierenden, wurden auch Angebote wie das „Munzinger-Archiv“, „Langenscheidt-Wörterbücher“ oder „Nationalsozialismus, Holocaust, Widerstand und Exil (Deutsche Geschichte im 20. Jahrhundert Online)“ gekauft, die eher dem landesbibliothekarischen Sektor zuzurechnen sind. Mit der Onleihe für den stadtbibliothekarischen Bereich schließt sich der Kreis und die HLB Fulda ermöglicht so auch den stadtbibliothekarisch interessierten Nutzerinnen und Nutzern im elektronischen Sektor einen Zugriff auf Volltexte unabhängig von Öffnungszeiten rund um die Uhr.

So wird die HLB Fulda ihrem Auftrag der umfassenden Medien- und Informationsversorgung in Fulda und der Region gerecht. Ob dies auch eine Lösung für andere wissenschaftliche Bibliotheken ist, kann nicht von Fulda aus beantwortet werden. Der Onleihe sind inzwischen neun weitere wissenschaftliche Bibliotheken beigetreten; in diesem Jahr sollen die nächsten folgen.

Wie geht es in Fulda weiter? Ein Schwerpunkt wird sein, den Ausbau des Medienangebotes zur Nutzung auf mobilen Endgeräten zu befördern. Es wird überlegt, ob nicht auch die entsprechende Hardware - also die Endgeräte - in der Bibliothek zum Verleih angeboten werden, wie es in anderen (öffentlichen) Bibliotheken schon Praxis ist. Dies wird auf einem anderen Gebiet schon vollzogen: Seit 2012 bietet die HLB Fulda die „Ting-Hörstift-Technologie“17 an und kaufte hier nicht nur die Bücher, sondern auch die entsprechenden Hörstifte und verleiht diese. Die Nachfrage danach gibt ihr Recht, auch mobile Endgeräte für die Nutzerinnen und Nutzer anzubieten.

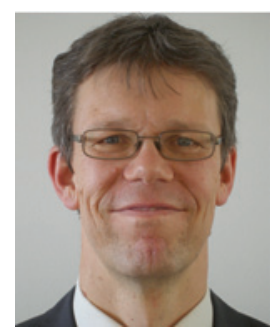

\author{
Berthold Weiß \\ Stellvertretender Leiter \\ Hochschul- und Landesbibliothek \\ Hochschule Fulda \\ Marquardstraße 35 \\ 36039 Fulda \\ Telefon: 0661/9640-9802 \\ E-Mail: berthold.weiss@hlb.hs-fulda.de
}

17 Neue Medien in der Stadtbibliothek: Lesen mit dem Hörstift sorgt für Aha-Effekte. In: Osthessen-News, 10.08.2012; URL: http://osthessen-news.de/n1217860/fulda-neue-medien-in-derstadtbibliothek-lesen-mit-dem-h-rstift-sorgt-f-r-aha-effekte.html. 